\title{
Research on the Implementation of Campus Football Policy in China from the Perspective of Comprehensive Model*
}

\author{
Yifeng Bu \\ Sports Institute \\ Jiangsu Normal University \\ Xuzhou, China
}

\author{
Luzhen Lai \\ Sports Institute \\ Jiangsu Normal University \\ Xuzhou, China
}

\begin{abstract}
Various factors of campus football policy implementation are analyzed by using literature research, logic analysis and other methods and combined with a series of policy documents and social environment of campus football, the Sabatir and Mazmanian policy implementation model. The study believes that the problem of campus football in China is complicated, the policy system is imperfect, the shortage of teachers and the lack of funds for implementation, the lack of linkage between the implementing agencies and the unclear responsibility, which makes low motivation and low recognition of the target groups, coupled with the unfavorable policy enforcement environment, and the supervision mechanism. And the evaluation system is not perfect, leading to policy implementation block. Therefore, we should change the ideological concepts of the target group, stimulate the internal motivation of the target group, clarify the implementation goals of the campus football policy, improve the supporting measures for policies, broaden the implementation resources of the policy, strengthen cooperation between the various executive departments, and establish a supervision system, improve the policy implementation mechanism and optimize the policy implementation environment. Thereby the efficiency of campus football policy implementation can be improved.
\end{abstract}

Keywords-campus football; policy implementation; Sabatir and Mazmanian comprehensive model

\section{INTRODUCTION}

In 2009, the General Administration of Sport and the Ministry of Education jointly issued the "Notice on Launching Youth Campus Activities" and the "Implementation Plan", marking the official launch of the campus football event. In March 2015, the General Office of the State Council issued the "Overall Plan for the Reform and Development of China's Football". Promoting the development of campus football has become the core task of football reform, and campus football activities have also risen to the national strategic level. However, campus football, as a pioneer of school sports reform, is different

*Fund project: 1. Research on Ecological Model Construction and System Optimization of Jiangsu Campus Football Development (17TYC004)

2. Campus Football Policy Implementation Study (2018YXJ356) from other general public affairs. It is embedded in the national education system and has certain complexity. Although the school football has achieved good results with the support of a good policy environment, it is still difficult to solve the fact that the practice of campus football is contrary to the actual situation. There are a series of problems such as "policy obstruction", "policy distortion" and "lack of individual initiative and participation in governance"(National Campus Football Special Research Report, 2018). American policy scholar Allison believes that in the process of achieving policy objectives, the program determines only $10 \%$ of the functions, and the remaining $90 \%$ depends on effective implementation. Therefore, it can solve the phenomenon that campus football practice is contrary to reality by improving the execution of campus football policy.

Through the CNKI search, since the school football activities in 2009, China's academic papers on campus football mainly focus on the investigation of current status, sustainable development, management system, training competition, talent cultivation, cultural connotation construction and value promotion. The policy implementation research that involves the most parameters and factors in the bottleneck of campus football development is still in its infancy.

\section{COMPREHENSIVE MODEL ANALYSIS OF POLICY IMPLEMENTATION}

\section{A. Theoretical Overview}

In 1979, Sabatir and Mazmanian of the United States proposed a comprehensive model for policy implementation. The biggest feature of this model is that it has extracted three major categories and seventeen factors that affect policy implementation, which is extremely comprehensive. The first category is the solvability of the problem: 1 . effective theoretical basis; 2 . diversity of the behavior of the target group; 3 . the number of target groups; 4 . the magnitude of the change in the behavior of the target group. The second category is the regulatory capacity of the policy itself: 1 . the policy itself contains sufficient causality; 2. clear policy directives; 3. adequate financial resources; 4. inter-agency 
and inter-agency level integration; 5 . the decision procedure of the actuator; 6. recruitment of executives; 7. opportunities for outsiders to participate. The third category is variables outside the policy: 1. economic environment and technology; 2. the degree of media attention to the problem; 3. public support; 4. attitudes and resources of interest groups; 5. support from the supervisory authority; 6. the executive's enthusiasm and leadership art.

\section{B. Model Import}

The integrated model of Sabatir and Mazmanian provides an important perspective for policy execution analysis and summarizes the factors that influence the various stages of policy implementation. In contrast to the implementation of China's campus football policy, the comprehensive model analysis generally covers the main aspects of its policy implementation. Furthermore, Sabatir and Mazmanian's comprehensive policy implementation model is a typical "top-down" policy implementation model, which is in line with China's policy reality — "the central government or superiors make policies, and local governments or subordinates implement policies". Therefore, it is feasible and appropriate to use the comprehensive model to analyze the elements of the implementation process of campus football policy.

\section{ANALYSIS OF FACTORS AFFECTING CAMPUS FoOtBALl UNDER THE COMPREHENSIVE MODEL OF POLICY IMPLEMENTATION}

\section{A. Characteristics of Policy Issues}

The effectiveness of policy implementation is first and foremost related to the type and nature of the policy issues to be addressed. The more complex the problem, the more difficult it is to perform.

1) Campus football is complicated and difficult to perform: short of the youth football population, the backwardness of football and the decline in the health of young people are important issues that need to be resolved on the road to becoming a sports power. As a pioneer of school sports reform, campus football must have certain complexity. It needs to take a long time to explore on the design of the football system, the penetration of culture, the investment of funds, the construction of venues, the professional football training and the nationwide popularity.

2) Target group cognition and the balance of interest: the target group's awareness of the policy, the degree of recognition and its own specific interests will seriously affect the implementation of the campus football policy. Mao Zhenming and others proposed that in order to establish a dynamic mechanism for campus football, we must solve the enthusiasm of principals and parents first [1]. Under the pressure of "Progression rate" and security accidents, some principals have weaker recognition of the campus football policy due to their political achievements. Some principals believe that the development of campus football is to establish several school football teams so that they can achieve excellent results in the inter-school competition. This is the dislocation of the principal's perception of policy. Parents from the children's career path, as well as the interests of life development, will also be opposed to the implementation of campus football. Even they know the value of campus football.

\section{B. Regulatory Capacity of the Policy Itself}

The key to restricting the effective implementation of Chinese campus football in the regulatory capacity of the policy itself is whether the policy directives are clear, whether the policy resources are sufficient, and whether the policy synergies between the implementing agencies are fully exerted

1) Campus football policy system is not perfect: although the campus football activities have achieved good results since the implementation, there are still many problems in the implementation of specific policies.

First of all, the legal effect is insufficient. In March 2015, the General Office of the State Council issued the "Overall Plan for the Reform and Development of China's Football", and the campus football activities also rose to the national strategic level. However, specific policies are issued in the form of administrative regulations and lower-level documents and notices, and their binding and legal effects are obviously insufficient. For example, the "Implementation Plan" requires that "all physical education teachers who participate in the campus football activity teacher training class will be awarded a training certificate after passing the training and examination, and will be included in the teacher continuing education time according to the corresponding time." However, the survey found that $64.5 \%$ of the schools did not implement this policy because of the lack of supporting guidance policies and corresponding institutional reforms, which made the incentives aimed at mobilizing teachers' enthusiasm become a mere scrap of paper [2].

Second, the policy content is formalized and vague. For example, the "China Football Reform and Development Overall Plan" emphasizes "improving the insurance mechanism, promoting the government to purchase services, improving the level of campus football safety and security, and lifting the worries of students, parents and schools"; "Improve the examination enrollment policy and allow the football specialties to move reasonably within a certain range when enrolling in higher education.". Regardless of whether it is an insurance mechanism or an enrollment policy, there is a lack of corresponding implementation rules and supporting measures. The supporting policies are not perfect and cannot fully play its guiding role in policy.

2) Campus football policy resources are seriously insufficient: Teacher resources, funding and venues are the key factors that constrain the effective implementation of China's campus football policy.

According to the survey of the campus football featured schools in 2018, the faculty of campus football is very short, 
showing the development trend of insufficient total quantity, low professionalism, unreasonable structure, unskilled training and regional imbalance. Due to the rapid development of campus football and the rapid increase in demand, the network survey data shows that there are 13,661 football majors in sports colleges, accounting for only $26.7 \%$ of the total. This indicates that the campus football professional talents are in short supply, and the training mode of football undergraduate professional talents cannot meet the development needs of campus football. On the other hand, the staffing of the education system and the constraints of talent recruitment conditions make it difficult for some football majors or retired football players to be admitted or transferred to the teacher series. There is limited funding for campus football. In 2013, the special funds for campus football were adjusted from 40 million to 56 million per year. However, after being allocated to football-specific schools, it is still a drop in the bucket. In 2015-2017, all provinces have strengthened the protection of campus football. The special funds invested through the financial and other channels have been increasing, but the number of football schools has been increasing, which has not increased the funding of football schools. The lack of funds is an important reason for the lack of football venues. In the 11 provinces surveyed by the Ministry of Education in 2018, there were 11,368 football fields, with an average of 0.967 per school, indicating that the basic venue was seriously inadequate.

3) Insufficient cooperation between implementing agencies and the responsibility is not clear: the "Opinions of the Ministry of Education and other 6 departments on accelerating the development of youth campus football" and the corresponding documents in various places have arranged tasks for the various departments of the campus work leading group. However, in the past three years, except for the education department, other departments have not The establishment of a clear list of responsibilities and tasks, so that the relevant departments at the grassroots level lack specific and specific compliance, so the policy synergy of multi-sector linkage has not been fully utilized at various levels. Especially from the end of 2016 to the beginning of 2017, after the Chinese Football Association was included in the national campus football leading group, the national youth campus football work leading group changed from the original 6 departments to " $6+1$ ", which made most provinces and cities fail to follow up in time. The education department and sports cooperation are weak, and the sports department, especially the football associations at all levels, fail to exert their talents and resources. The National Youth Campus Football Work Leading Group Office does not have full-time staff. Many local campus football work leading group offices are not set up. Most of the staff are part-time, heavy work, long tasks so that the quality is not guaranteed.

\section{Variables Outside the Policy}

Sabatir and Mazmanian believe that in the comprehensive model of policy implementation, variables outside the policy also have an important impact on policy implementation.

1) Economic environment constraints, lack of cultural environment: in terms of economic environment, there are fewer sources of funding for campus football development, mainly from state funding, and the state-specific funds are limited, requiring financial support from all levels of government. Due to the economic differences between the east and the west, the development of campus football is not balanced and the ecological construction is not synchronized. In terms of cultural construction, the campus football classroom teaching, extracurricular training, campus competition, and cultural activities are not fully developed, which seriously affects the popularity and level of campus football. In addition, in order to achieve rapid results, the grassroots departments and schools are paying more attention to the organization or participation of all levels of campus football competitions, and use limited resources for a small number of students participating in competitions at all levels and abdicate the attention and investment in the football culture activities that most students can participate in restricts the overall popularity of campus football.

2) The supervision mechanism and evaluation system are not perfect: the National Youth Campus Football Leading Group has issued a series of rules and regulations, forming a set of working mechanisms to promote campus football work, but in addition to the "National Campus Football Characteristic School Basic Standards (Trial)", There are no evaluation index systems, management information systems and quality monitoring systems at different levels. For the operation and connection of all levels of campus football matches, the administrative staff and public servants involved in the campus football management work, the teachers, coaches and referees engaged in the campus football teaching and training competition have supervision, inspection and investigation. However, they failed to establish the review standards, scientific supervision and campus football work in all levels of educational supervision indicators. Due to the lack of such a policy implementation mechanism and the lack of monitoring mechanisms, the operability is poor in practice, resulting in the failure of policies in implementation [3].

\section{SUGGESTIONS FOR IMPROVING THE IMPLEMENTATION OF CAMPUS FOOTBALL POLICY}

\section{A. Changing the Idea of the Target Group and Stimulating the Internal Motivation of the Target Group}

The campus football policy is ultimately achieved through the recognition of policies and compliance by principals, parents, and students [4]. Schools with weak campus football, both teachers and parents, have not talked more about the educational functions of football. They only consider football around cultural achievements and student safety, and they do not realize the significance of full 
success behind football. Wang Dengfeng, director of the Department of Sports Health and Art Education of the Ministry of Education, pointed out that compared with funds, teachers and venues, the biggest bottleneck in the development of campus football is still people's ideas. Therefore, in the future training of campus football, we should gradually increase the training of principals and teachers in the areas where campus football development is lagging, and put the core concept of campus football education into their education and teaching activities, so that these concepts can be spread to parents. Only in this way, will the development of campus football have lasting motivation.

Campus football should implement the purpose of educating people first, and learn from the development concept of campus football in developed countries. For example, Germany, France, and the United Kingdom clearly stipulate that participation in football training cannot affect the study of normal culture classes; students from the United States and Japan who fail to pass the cultural scores stop training and suspension [5]. Improve the examination system, incorporate football into the exam-required examinations, open up the pathway for further studies, and establish a pathway for the transportation of campus football talents from "primary school-junior high school-high school-university" [6]. Improve the comprehensive insurance system for campus injury, which can solve the worries of parents.

\section{B. Clarifying the Implementation Goals of the Campus Football Policy and Improving the Policy Supporting Measures}

In the process of policy implementation, it is necessary to use the benchmark of policy objectives to measure various stages of policy implementation, and to prevent the phenomenon of alienation and blockage in the process of implementation. The alienation of campus football policy implementation is essentially the alienation of goals. Its types can include alienation of rights and responsibilities, alienation of participating entities, and alienation of public opinion, which are the root causes of various confusions and dislocations [7]. It is necessary to define and carefully sort out the theory and positioning, and carry out accurate analysis and publicity, and strengthen the guidance and supervision of campus football from understanding and implementation. The government should improve the supporting measures of the policy, improve the supporting policies, and give full play to the guiding role of the policy. Due to the variety of situations facing the development of campus football in various places, the problems encountered vary widely. All provinces and cities should adapt to local conditions, further clarify and operate relevant national documents, and introduce a policy support system that is in line with their own characteristics.

\section{Broadening Resources for Policy Implementation}

The shortage of campus football teachers and the lack of implementation funds have seriously affected the effective implementation of policies. It is necessary for the competent authorities at all levels to issue more relevant and relevant supporting policies, improve the compensation mechanism, learn from the advanced management experience of the United States and Germany, and encourage market institutions to sponsor campus football through tax incentives to reduce the government's financial expenses [8]. All localities should incorporate the construction of campus football activities into the overall plan for school construction in accordance with the requirements of the "Implementation Opinions". In accordance with the principle of adapting to local conditions and gradually improving, through various ways such as building new sites, rebuilding existing sites, renting social sites, and sharing community sites and coordinating the problem of insufficient facilities for campus football venues. In view of the current shortage of football teachers, all localities should further broaden the channels for schools to match and complement football teachers, increase the recruitment of football teachers, and encourage other teachers with football specialties to take part in football classes. Football coaches, referees and trained excellent football retired athletes as well as volunteers with football expertise are constantly encouraged to serve as part-time teachers to enrich the campus football faculty.

\section{Strengthening Horizontal Linkage and Vertical Communication Among Departments}

Since there are some places in the provinces where campus football management is not in place or inefficient, it is necessary to solve the institutional settings of the leading groups at all levels from the policy level. Full-time staff should be equipped to be responsible for the planning, management, guidance, inspection and evaluation of campus football, and stipulate corresponding job responsibilities and performance requirements, so that the standing leadership of campus football can really play its due role. The implementation of the campus football policy requires the cooperation of various government departments. Each department should have a clear list of responsibilities and a list of tasks, so that relevant departments at the grassroots level can have a basis for doing this work and have evidence to follow. At all levels, it is necessary to give full play to the policy synergies of multi-sectoral linkages and resolutely avoid mutual "interest barriers" arising from the powers conferred by the functions of the functional departments [9]. Especially between the sports department and the education department, the sports department has excellent coaches, football talents and venue facilities, and strengthens the connection between the sports department and the education department, so that high-quality sports resources are shared with school education, thereby ensuring the smooth development of campus football activities. 


\section{E. Establishing a Supervision System and Improving the Policy Implementation Mechanism}

If the effective implementation of the policy is hindered, it is necessary to improve the enforcement of the policy through a mandatory institutional system [10]. For the effective implementation of the campus football policy, it is necessary to establish a policy implementation supervision and management mechanism. The National Youth Campus Football Work Leading Group should incorporate campus football into the education supervision index system, formulate a special supervision method for campus football, and establish a feedback mechanism through regular special supervision, irregular special inspections, compulsory event participation, and special investigations at various levels. Strengthening the management of the target, mobilizing the sense of responsibility and mission of all personnel involved in the campus football work are very important, which can gradually form a supervision system to make the working mechanism more scientific and efficient.

Football is a sport that is more vulnerable to injury, and China's sports liability insurance is relatively simple. In contrast, Japanese campus football insurance is not only a wide variety, but also covers a wide range. The first thing that schools pay after tuition fees for students is to purchase insurance for student players [11]. To this end, we should actively explore and improve the comprehensive insurance system for campus injury, so that parents can safely encourage their children to play football. At the same time, the incentive mechanism should be improved, the enthusiasm of the executive authorities and implementers should be improved, and the implementation of the policy plan should be encouraged [12].

\section{F. Optimizing the Policy Implementation Environment}

Due to the existence of economic differences, in the context of balanced education, special attention should be paid to the characteristic development of schools in povertystricken areas, and support policies for poverty-stricken areas should be introduced. Differentiate and refine the amount of special and matching funds, the source and scope of funds, the use and methods of expenditure, and other related aspects of campus football, especially to address the current needs of campus football in poor areas. All parts of the country should actively promote the construction of campus football culture, enrich football culture activities, and infiltrate the campus football culture into the daily teaching and life of schools and students. The first is to integrate football elements into the cultural construction of the campus. The second is to include football in the big class and extracurricular sports activities, enrich the content of one hour of sunshine sports activities on campus every day. The third is to integrate football activities into student life, build a campus football team and a football interest group, and constantly inspire students to participate in campus football's enthusiasm and passion, let the children know the football, love the football. The publicity of campus football activities should be strengthened to let more people know about campus football, understand campus football and watch campus football league, which can attract commercial sponsorship from social enterprises, reduce financial pressure from the government and create an excellent environment for the development of campus football.

\section{CONCLUSION}

In view of China's political system and management model, the comprehensive model of Sabatir and Mazmanian policy implementation can better analyze the status quo and problems of China's campus football policy implementation. The analysis found that the complexity of the campus football policy, the unclear level of objectives, the lack of policy implementation resources, the imperfect implementation mechanism, the lack of linkage between the implementing agencies, the lack of motivation of the target group, and the unfavorable implementation environment are all important factors restricting the implementation of the campus football policy. Therefore, in the process of implementing the campus football policy, the government should clarify the policy implementation objectives, improve the implementation mechanism of the policy, broaden the implementation resources of the policy, strengthen cooperation between the executive agencies, optimize the environment for policy implementation, and improve the recognition of the target group so that the campus football policy can be implemented effectively.

\section{REFERENCES}

[1] Mao Zhenming, Liu Tianqi, Yan Liuhong. On the Top Design of "New Campus Football"[J]; Journal of Wuhan Institute of Physical Education, 2015-03.

[2] Li Jixia, He Zhilin, Dong Zhongming, $\mathrm{Xu}$ Yangcai. On the Development Bottleneck and Its Breakthrough in National Youth School Football Activity[J]; Journal of Shanghai University of Sport; 2012-03.

[3] Yang Chengwei, Tang Yan, Zhang He, Zhang Hong. Effective Execution Path of Adolescents' Physical Health Policy - Based on the Perspective of Mitt-Horn's Policy Implementation System Model[J]; Sports Science, 2014-8.

[4] Jiangnan. The Restrictive Factors and Path Choice of Campus Football Policy Implementation in China — Based on the Perspective of Smith's Policy Implementation Process Model[J]; China Sports Science and Technology, 2017-01.

[5] Zhang Ting'an. Carrying out the campus football activities requires the concept to lead [J]; Journal of Beijing Sport University, 2015-08.

[6] Zhang Yuan, Zhang Ting'an. Research on the Implementation Strategy of Campus Football Policy in China[J]; Sports Culture Guide, 2018-05.

[7] Li Xinwei, Li Wei. The Alienation of Campus Football in China[J]; Journal of Physical Education, 2015-05.

[8] Qiu Lin1, Dai Fuxiang, Zhang Ting'an. Research on the Positioning of Government Functions in the Development of Campus Football in China[J]; Journal of Wuhan Institute of Physical Education, 2016-06.

[9] Zhang Ting'an. The Scientific Outlook on Development Should Be Established in the Future Development of Campus Football in China [J]; Journal of Beijing Sport University, 2015-01.

[10] Yang Chengwei, Tang Yan. Implementation Dilemma and Path Optimization of School Sports Facilities Service Social Policy[J]; Journal of Physical Education, 2013-06. 
[11] Wang Yulin, Wang Li, Kang Huibin. Comparative Study on the Development of Chinese and Japanese Youth Campus Football[J]; Journal of Xi'an Institute of Physical Education, 2014-06.

[12] Li Weidong. Research on Football Competition System at School in China, Japan and South Korea[J]; Journal of Beijing Sport University, 2013-10. 